\title{
Application of 2D and 3D Optical Microscopy in the Examination of Suspect Counterfeit Pharmaceutical Tablets
}

\author{
S. Frank Platek, Nicola Ranieri, Douglas C. Albright and Mark W. Witkowski \\ US Food and Drug Administration - Forensic Chemistry Center, Trace Examination Section \\ 6751 Steger Drive, Cincinnati, Ohio 45237-3097
}

One of the areas of research and case analyses performed at the US Food and Drug Administration's Forensic Chemistry Center (FCC) is the identification of counterfeit pharmaceutical tablets. A final step in the pharmaceutical tablet manufacturing process includes compression of the tablet powder formulation. The compress involves using a tablet die and two opposing punches which apply any debossed character(s) and/or score lines to the tablet face. Tablet punches can be precision manufactured to very high tolerances using tablet diagram engineering drawings. Counterfeit tablets may or may not have been made with high quality punches making analysis of tablet debossing an important examination. This examination may include debossed character font, size, placement, and depth of debossed characters, etc. Irregularities and non-conformities between the counterfeit tablets and the authentic tablet debossing and authentic tablet punch engineering diagrams may provide important information in the identification and possible source of a counterfeit tablet.

The FCC's Trace Examination Section uses a variety of microscopic applications including stereoscopic (SLM), polarized (PLM), and scanning electron (SEM) microscopy, along with mid-infrared, Raman and Energy Dispersive X-Ray spectroscopy. Image analysis (IA), and most recently, the use of surface profilometry / 2D and 3D optical microscopy are being used to examine pharmaceutical tablet coating, core and debossing characteristics.

\section{D Tablet Face Analysis:}

The FCC uses 2D image overlay comparison analyses to differentiate counterfeit tablets from authentic tablets. 2D imaging involves using a SLM coupled with high resolution camera and image analysis software to capture and compare tablet faces. Image capture and analysis of the suspect tablet debossing when overlaid with an authentic tablet image can demonstrate differences in tablet debossing (FIG 1). IA along with other physical and chemical analyses permits a complete comparison of suspect counterfeit to authentic pharmaceutical products.

\section{D Tablet Face Analysis:}

A recently acquired 3D surface profilometry microscope is showing great promise in the examination of surfaces including tablet debossing (FIG 2) as a quantifiable analysis method. [1] The principle of this technique combines a precise depth of focus measurement in relation to the precise location on the tablet which provides topographical and color information from the variation of focus. A proprietary algorithm reconstructs these measurements into a single 3D data set with highly accurate topographical information (FIG 3). The high vertical resolution $(\sim 1 \mu \mathrm{m})$ measurements of suspect tablet face features are made and then compared with measurements made on authentic tablet faces over the same region. A plot of the measured pixel by pixel values over a selected debossed surface (usually a line profile across the tablet face) from both suspect counterfeit and authentic tablets is compared (FIG 4). Traceable calibration standards allow the verification of measurement results. System validation is currently in progress using tablets of known authenticity which are compared to known counterfeit tablets. In the example shown, the surface measurements from one authentic tablet (A) were overlaid with surface measurements from one known counterfeit tablet (C). The results were consistent with a suspect tablet that was not produced with tablet punches used to produce the authentic tablet.

References

[1] H.Schroettner et al., Microchim Acta 155, (2006) 

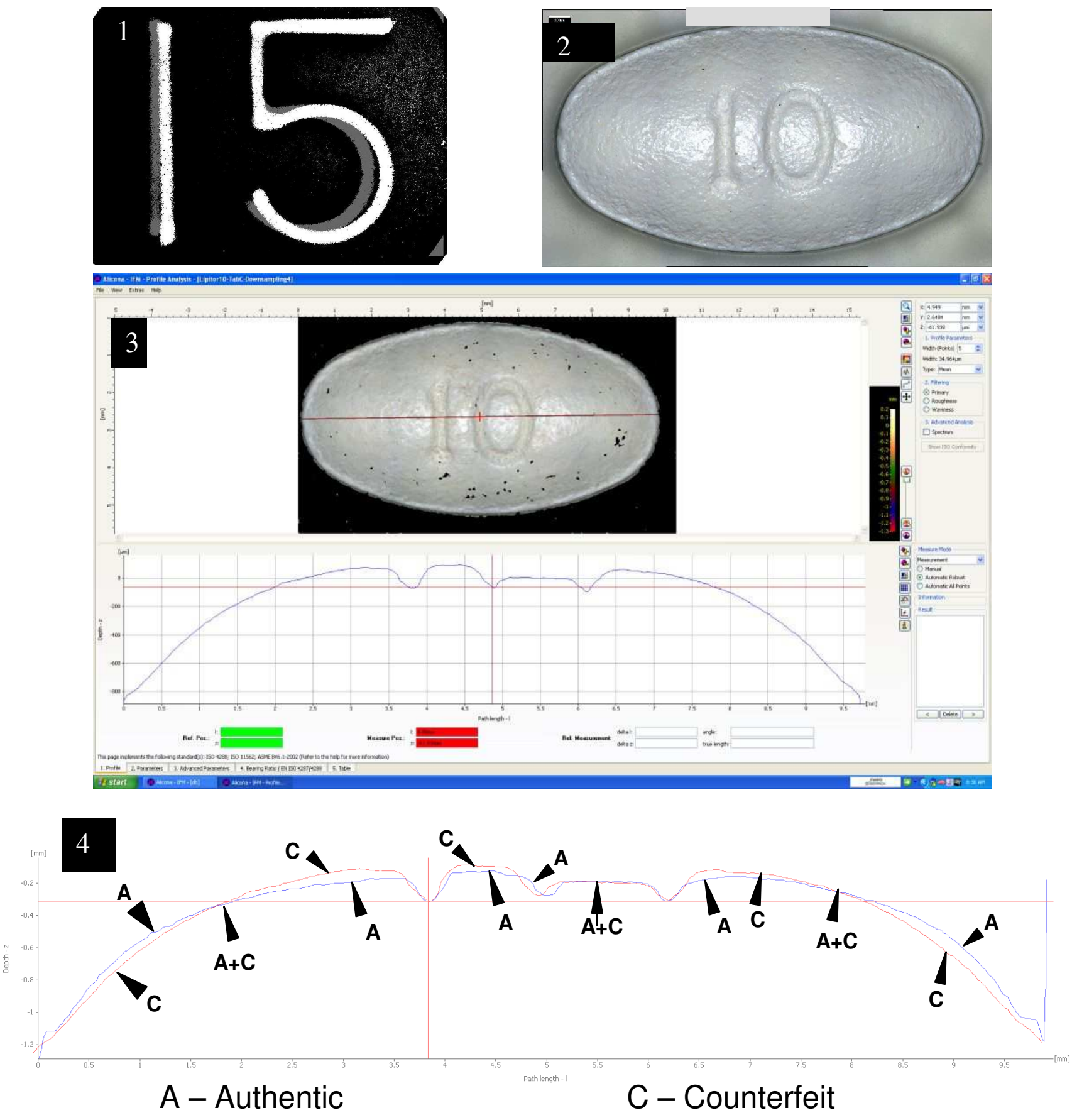

FIG. 1. SLM IA overlay of 2D images of tablet debossing (authentic vs. known counterfeit)

FIG. 2. Example of tablet face with debossed "10"

FIG. 3. Example of quantitative measurements across a debossed tablet face

FIG. 4. Overlaid surface measurement graphs from a representative authentic debossed tablet face (A) and a known counterfeit debossed tablet face (C). 